\title{
Three-dimensional phase-field study of crack-seal microstructures - insights from innovative post-processing techniques
}
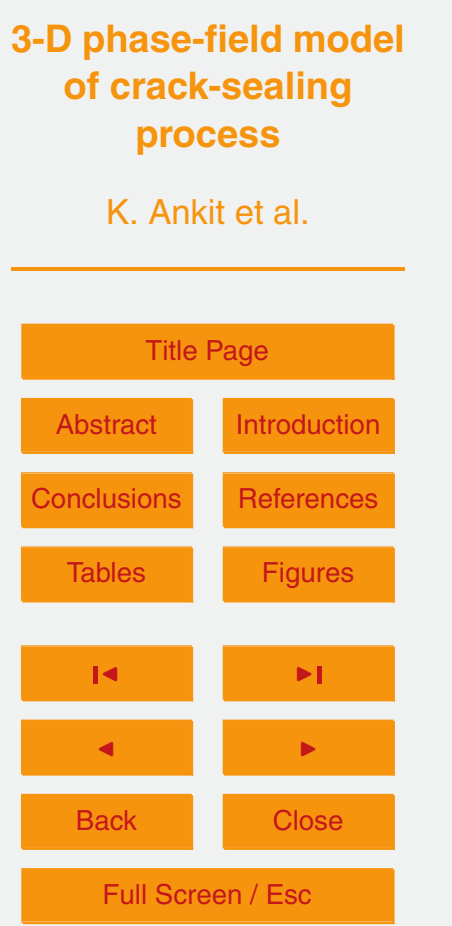

Published by Copernicus Publications on behalf of the European Geosciences Union.

Printer-friendly Version

Interactive Discussion 


\section{Abstract}

Numerical simulations of vein evolution contribute to a better understanding of processes involved in their formation and possess the potential to provide invaluable insights into the rock deformation history and fluid flow pathways. The primary aim of

5 the present article is to investigate the influence of a "realistic" boundary condition, i.e. an algorithmically generated "fractal" surface, on the vein evolution in 3-D using a thermodynamically consistent approach, while explaining the benefits of accounting for an extra dimensionality. The 3-D simulation results are supplemented by innovative numerical post-processing and advanced visualization techniques. The new methodologies to 10 measure the tracking efficiency demonstrate the importance of accounting the temporal evolution; no such information is usually accessible in field studies and notoriously difficult to obtain from laboratory experiments as well. The grain growth statistics obtained by numerically post-processing the 3-D computational microstructures explain the pinning mechanism which leads to arrest of grain boundaries/multi-junctions by crack peaks, thereby, enhancing the tracking behavior.

\section{Introduction}

Correct interpretation of vein microstructure demands careful study of thin sections and appropriate bridging of results obtained from different length scales. The field based studies as well as laboratory experiments are the two different means by which geologists try to establish structure-property co-relationship. Unless one performs arduous in-situ laboratory experiments, the time-based evolution information stays inaccessible. Moreover, it is difficult to decompose the effect of different processes that might have acted in sequence or simultaneously. Piazolo et al. (2010) summarize the limitations of field based studies and laboratory experiments while highlighting the general capability
GMDD

$7,631-658,2014$
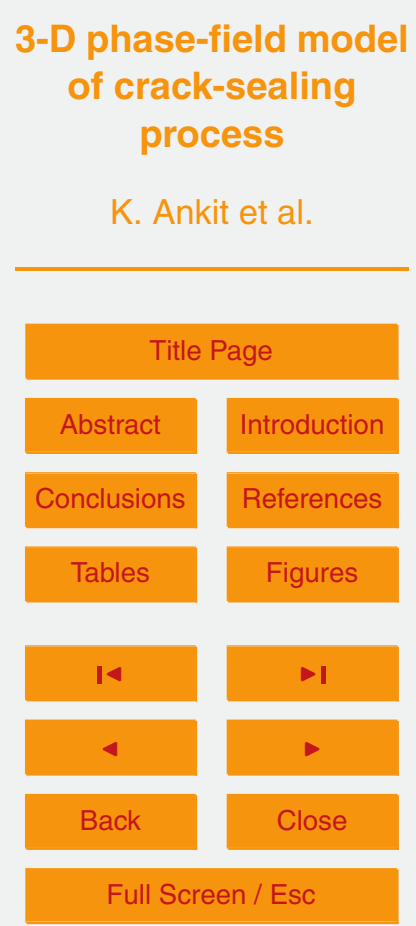

Printer-friendly Version

Interactive Discussion 
The need for numerical simulations to study microstructural evolution in veins has lead to development of computer programs, based on front-tracking approaches, like Vein Growth (Bons, 2001) and FACET (Zhang and Adams, 2002) in the past and more recently, Elle (Jessell et al., 2001; Bons et al., 2008). A major limitation of such front5 tracking methods is that numerical studies require huge effort to be extended to 3-D. The idea of comparing the 2-D numerical results with thin sections of 3-D natural samples is far-fetched, primarily because of an added degree of freedom for the evolving crystals (in 3-D) which leads to erroneous interpretation. While Bons (2001) provide hints concerning deviations when thin sections are directly compared with 2-D simu10 lation results, the actual differences remain unclear due to lack of any 3-D numerical results. It is noteworthy, that a complete understanding of the vein growth mechanism cannot be achieved by merely studying thin sections as it reveals only a part of the whole story. The partial story could often be misleading, since what looks to be a crystal not tracking the crack-opening trajectory in 2-D microstructure may turn out to be exactly opposite when studied in 3-D. In the context of vein growth, we emphasize that the grain formation process is generically of 3-D nature and can be interpreted in a physically sufficient manner by methods capable of capturing the growth characteristics and dynamics in full 3-D space. The phase-field simulation example shown in Fig. 1 bear testimony to our assertion.

20 The last two decades have seen the emergence of phase-field method as a versatile and popular tool for numerical simulations of crystal growth and in general, for a variety of other moving boundary problems; prominent application areas being solidification, solid-state phase transformations like spinodal decomposition and crack propagation. The most significant computational advantage of a phase-field model is that explicit tracking of the interface is unnecessary. In simpler words, in a phase-field simulation, freely moving interfaces between different phases or crystals (grain orientations) do not appear as geometric boundaries, i.e. places at which boundary conditions have to be applied explicitly. Instead, all the information about the location of the phase boundaries is implicitly contained in the phase-field variable, which obeys a partial differential
GMDD

7, 631-658, 2014
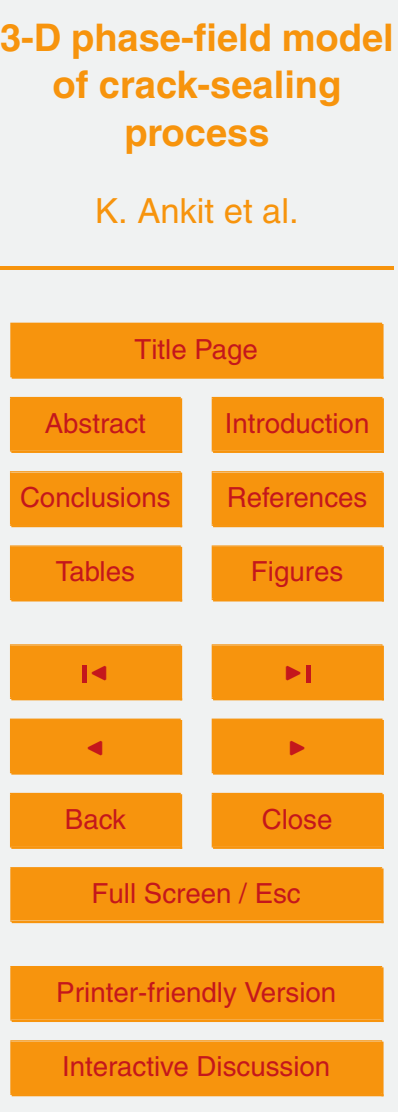
equation and solved within the entire computational domain. Different thermodynamic driving forces for topological changes, such as chemical bulk free energy, interfacial energy, elastic strain energy and different transport processes, such as mass diffusion and advection, can be coupled, thereby facilitating the comprehensive studies of the 5 transformation phenomena.

Hubert et al. (2009) discuss the limitations of Vein Growth and FACET (geometric restrictions and extension to $3-D$ ) and introduce a phase-field model to study crystal growth in veins, which uses a non-faceted anisotropy for the interfacial energy. However, it is well known from literature (Taylor and Cahn, 1998) that smooth continuous 10 functions cannot be used to simulate crystals with flat facets and sharp corners. Ankit et al. (2013b) show the importance of adopting a general thermodynamically consistent approach in modeling the evolution of vein microstructures by considering faceted-type anisotropy formulations of the interfacial energy function to simulate crystals with flat facets and sharp corners. Various boundary conditions and parameters which influence the crystal growth mechanism in veins, especially the grain boundary tracking behavior, can be successfully investigated using the phase-field method. Further, the reproducibility of previous simulation results (from front-tracking models) as well as chief advantages of adopting the novel multiphase-field model, such as 3-D numerical studies for crystal of any shape, large-scale simulations with many thousand grains and provision to implement transport, is highlighted.

In the current article, we advance the 3-D numerical study of Ankit et al. (2013b) to study the influence of a realistic representation of the crack wall by a boundary condition of fractal shape, on crystal growth in crack-sealing veins. By employing advanced visualization and innovative post-processing techniques, new methodologies to calculate "general tracking efficiency" for a more complex motion of grain boundaries is proposed. The present work highlights the importance of accounting the time evolution rather than calculating tracking efficiency solely based on final grain boundary morphology. Further, grain statistics such as temporal evolution of the number of tracking veins and the corresponding orientation and size distribution is obtained from the
GMDD

7, 631-658, 2014
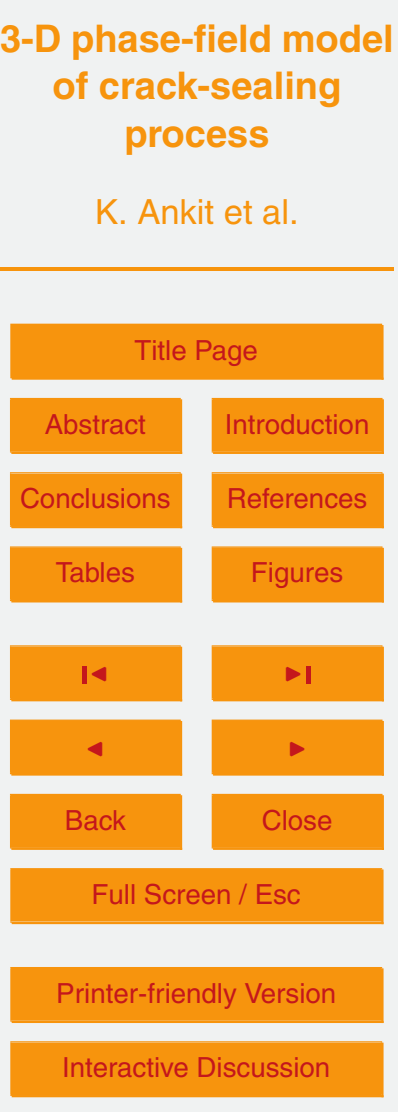
present large scale 3-D phase-field simulations with an aim to relate the shift in growth mechanism as a function of crack-opening rate, which is missing in previous numerical studies.

\section{Methods}

\subsection{Phase-field model}

The foundation of multiphase-field method utilized to address crystal growth problem is realized by a Helmholtz free energy functional formulated as

$\mathcal{F}(\boldsymbol{\phi})=\int_{\Omega}\left(f(\boldsymbol{\phi})+\varepsilon a(\boldsymbol{\phi}, \nabla \boldsymbol{\phi})+\frac{1}{\varepsilon} w(\boldsymbol{\phi})\right) \mathrm{d} x$,

where $f(\phi)$ is the bulk free energy density, $\varepsilon$ is the small length scale parameter related to the interface width, $a(\phi, \nabla \phi)$ is the gradient type and $w(\phi)$, a potential type energy density. The phase-field parameter $\boldsymbol{\phi}(\boldsymbol{x}, t)=\left(\phi_{1}(\boldsymbol{x}, t) \cdots \phi_{N}(\boldsymbol{x}, t)\right)$ describes the location of " $N$ " crystals with different orientation in space and time. The evolution equations for the phase-field vector components are described by variational derivative of the free energy functional which ensures energy and mass conservation as well as an increase

$\tau \varepsilon \frac{\partial \phi_{\alpha}}{\partial t}=\varepsilon\left(\nabla \cdot a_{, \nabla \phi_{\alpha}}(\boldsymbol{\phi}, \nabla \boldsymbol{\phi})-a_{, \phi_{\alpha}}(\boldsymbol{\phi}, \nabla \boldsymbol{\phi})\right)-\frac{1}{\varepsilon} w_{, \phi_{\alpha}}(\phi)-f_{, \phi_{\alpha}}(\boldsymbol{\phi})-\lambda$

The symbol $\tau$ is the kinetic coefficient and comma separated sub-indices represent derivatives with respect to $\phi_{\alpha}$ and gradient components $\frac{\partial \phi_{\alpha}}{\partial \chi_{i}}$. The lagrange multiplier $\lambda$ guarantees the summation constraint $\left(\sum_{\alpha=1}^{N} \phi_{\alpha}=1\right)$. The multiphase-field model equations and numerical implementation have been previously discussed in detail
3-D phase-field model

of crack-sealing

process

K. Ankit et al.

\section{Title Page}

Abstract

Introduction

Conclusions

References

Tables

Figures

14

$\rightarrow 1$

4

Back

Close

\section{Full Screen / Esc}

Printer-friendly Version

Interactive Discussion 
(Nestler et al., 2005; Stinner et al., 2004). The phase-field evolution Eq. (2) is nondimensionalised to ensure numerical accuracy during the computation. A brief account of the non-dimensionalisation procedure is provided by Wendler et al. (2009).

In the present work, we adopt the approach of Ankit et al. (2013b) to construct 5 the interfacial energy function $a(\phi, \nabla \phi)$ for a geologically relevant mineral "quartz". The polar-plot of interfacial energy and the corresponding equilibrium crystal shape is shown in Fig. $2 a$ and $b$, respectively. The equlibrium crystal shape represents an idealized quartz crystal consisting of $\{10 \overline{1} 0\},\{10 \overline{1} 1\}$ and $\{01 \overline{1} 1\}$ facets. An implicit assumption of the numerical simulations of vein growth process, presented in the later 10 sections of this article is that the smaller crystal facets $(\{2 \overline{1} \overline{1} 1\}$ and $\{6 \overline{1} \overline{5} 1\})$ possess higher growth rate and hence, do not influence the final polycrystalline morphology. Therefore, such faster growing and smaller facets are ignored in the present numerical studies. However, it is to be noted that it is possible to simulate such facets, provided the polar-plot of interfacial energy (cusps) accounts for them. Hence, the assumption made in present work should be interpreted as shape simplification and not as a limitation of the simulation algorithm.

\subsection{Numerical aspects}

With an objective to numerically simulate the crack-sealing process and to characterize the resulting microstructure, we choose quartz crystals as a representative vein forming material. An algorithmically generated (diamond-square algorithm) fractal surface is utilized to model a three dimensional crack surface (boundary condition) for phasefield simulations as shown in Fig. 3.

The progressive splitting of host-rock and crystal precipitation in the open space is algorithmically replicated once by numerical pre-processing to obtain the initial con25 dition for simulation as shown in Fig. 4. The preprocessing algorithm adopted is as follows:
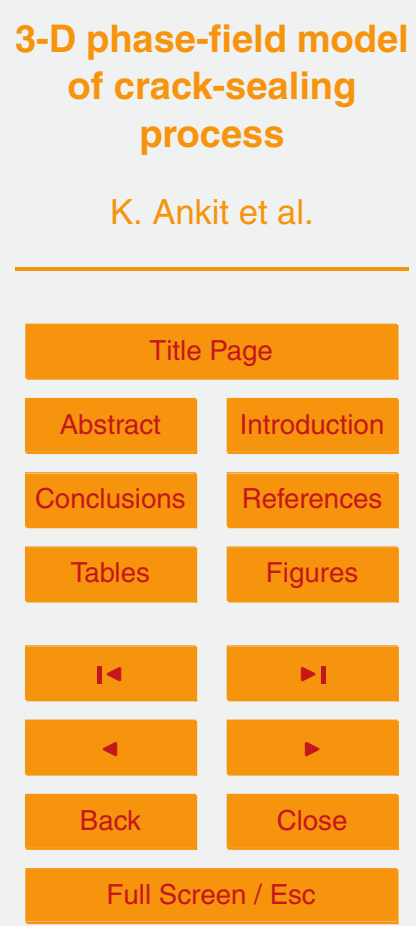

Printer-friendly Version

Interactive Discussion 
- The fractal topology of the lower crack surface is generated by a C program to implement the well-known diamond-square algorithm (Miller, 1986). The heightmap of upper crack surface is obtained by subtracting the respective heights (for lower crack-surface) from the total height of the parent rock in consideration. The two complementary fractal surfaces are stationed over each other with a minor clearance which represents the fracture in host rock.

- The space between the upper and lower surface is increased (by 5 grid points in the splitting direction) which represents the first crack-opening event.

- Crystals of equal size with different orientation in space are initially laid on the lower crack surface. At this point, it is important to note that the size of the crystal nuclei needs to be equal in order to negate the advent of size-effects in the following vein growth simulations. The numerical pre-processing technique in order to rule out any such possibilities involves the following sub-steps: cuboid crystal nuclei (different colors represent an axial tilt defined in the previous section) are generated separately and merged with the parent numerical domain containing the cleft (a boundary condition) such that the former can be over-written. The resulting domain can be described as a perfectly sealed microstructure.

- The lower crack surface is shifted downwards again to create a small space between the wall and crystal front. Thus, we obtain the initial numerical domain to start the phase-field simulations.

We simulate the unitaxial opening and sealing of cracks to investigate the formation of 3-D crack-sealing microstructure using the initial condition generated in Fig. 4, and thereby, characterize the tracking behavior of grain boundaries and multi-junctions. The following discussion will focus on the two 3-D simulation test cases with the same initial condition but different crack-opening rate, as described in Table 1. The phase-field evolution Eq. (2) is solved numerically using an explicit forward Euler scheme. The spatial derivatives of the right hand side equation are discretized using a second order
GMDD

7, 631-658, 2014
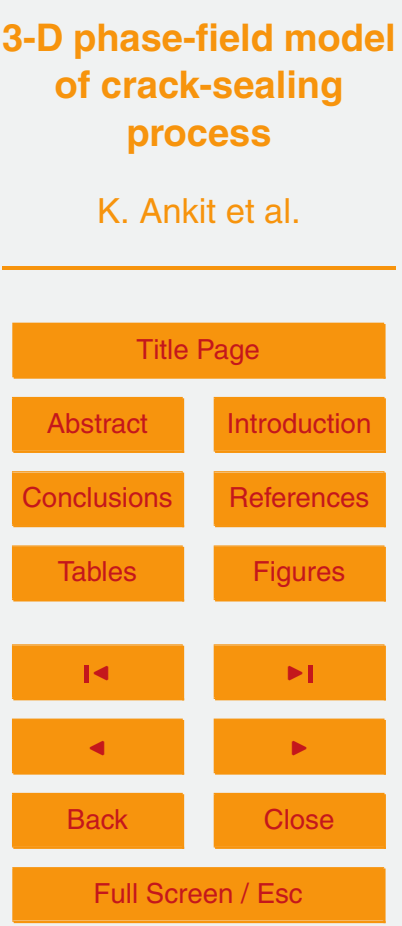

Printer-friendly Version

Interactive Discussion 
accurate scheme with a combination of forward and backward finite differences. The implementation of a locally reduced order parameter optimization employs a dynamic listing of a limited number of locally existing grains and enable to reduce computation time so that large scale simulations in 3-D become feasible. The phase-field simulations 5 are performed on Linux high-performance computation clusters using a $\mathrm{C}$ program with parallel algorithms for domain decomposition to distribute the computing task on different nodes.

As a result of wall rock opening along the predetermined opening trajectory, the crack-aperture increases during simulation run-time. This adversely affects the computational efficiency as the size of simulation domain increases in the splitting direction. In order to avoid such complications, the simulation is carried out in a moving frame (also known as shifting-box simulation). In the present simulations, the domain is shifted in the growth direction (downwards) by adding a row of grid-point at the top of domain and discarding off a row of grid-points at the bottom, every time the advancing crystal growth front fills up $10 \%$ of the simulation box. The final domain is obtained by aggregating back the discarded rows of pixels as described by Ankit et al. (2013a) for a different material system. Further, we ensure that the advancing crystal-rock interface always stay within the boundaries of shifting-box for every simulation time-step.

\section{Simulation results}

\subsection{Calculation of tracking efficiency}

The term "tracking efficiency" which was first introduced by Urai et al. (1991) is frequently used to quantify the tracking behavior of crack sealing microstructures. Ankit et al. (2013b) amend this definition to a "general tracking efficiency" (GTE) based on a fitting procedure for linear crack opening trajectory. It is to be noted that both the above definitions of grain boundary tracking efficiency are based on the final microstructure morphology and do not account for the temporal evolution of grain
GMDD

7, 631-658, 2014
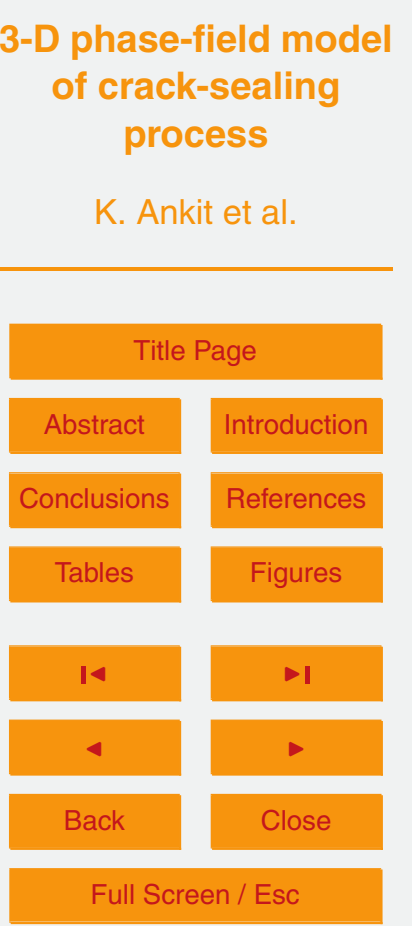

Printer-friendly Version

Interactive Discussion 
boundary tracking behavior. The dynamics of tracking efficiency is particularly important when the wall roughness of the advancing crack surface is not sufficiently high and the opening trajectory is non-linear. For such cases, general tracking efficiency is numerically obtained by fitting a straight lines in infinitesimally small time interval $\delta t$ in 5 which both the crack opening as well as grain boundary morphology can be assumed to be linear. In the following section, we highlight the advantage of calculating tracking efficiency by accounting for time evolution of grain barycenter. We calculate the tracking efficiency of the 3-D computational microstructure by two different methods:

1. The iso-surface of surviving crystals which are in contact with the advancing wall rock is visualized and the local peaks (10 nearest neighbors approximation) of the facing fractal surface are plotted. The peaks of the fractal surface lying along the grain boundaries/multi-junctions are extracted from the computational microstructure of the simulations $A$ and $B$ and are then superimposed, as shown in Fig. $5 a$ and $\mathrm{c}$, respectively. The total number of the grain boundary/multi-junction tracking peaks are designated as $N_{\mathrm{tp}}$. Similarly, those fractal peaks which neither lie along grain boundaries nor at multi-junctions are plotted in Fig. $5 \mathrm{~b}$ and d. The total number of such non-tracking peaks is denoted by $N_{\text {ntp }}$. The general tracking efficiency (GTE) for the 3-D crack-seal microstructure is defined for the $i$ th simulation time-step as:

$\mathrm{GTE}_{1}^{i}=\frac{N_{\mathrm{tp}}^{i}}{N_{\mathrm{ntp}}^{i}}$.

The definition of $\mathrm{GTE}_{1}^{i}$ is extended to account for the overall temporal evolution of tracking efficiency as:

$\mathrm{GTE}_{1}=\frac{\int_{0}^{t} \frac{N_{\mathrm{tp}}^{i}(t)}{N_{\mathrm{ntp}}^{i}(t)} \cdot \mathrm{d} t}{t}=\frac{\sum_{i=1}^{n(\delta t)} \frac{N_{\mathrm{tp}}^{i}}{N_{\mathrm{ntp}}^{i}} \cdot(\delta t)}{n(\delta t)}=\frac{\sum_{i=1}^{n(\delta t)} \frac{N_{\mathrm{tp}}^{i}}{N_{\mathrm{ntp}}^{i}}}{n}$
GMDD

$7,631-658,2014$

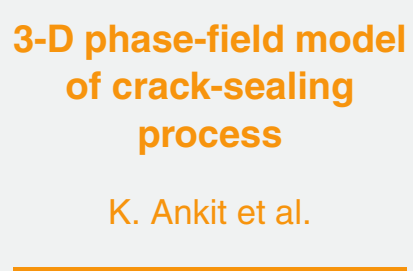

Title Page

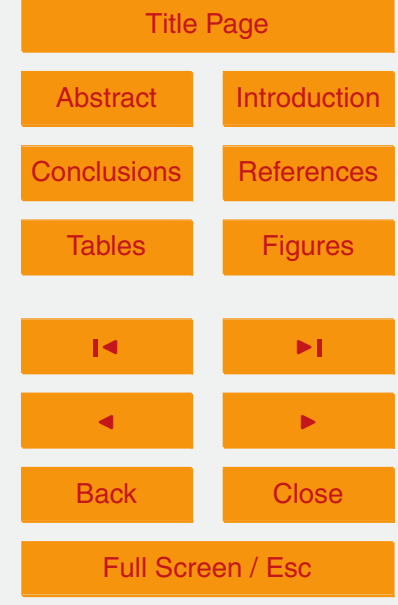

Printer-friendly Version

Interactive Discussion 
where $\delta t, n$ and $t$ represent time-step width, number of time-steps and total simulation time respectively.

2. The second definition of grain boundary tracking efficiency takes into account the temporal evolution of barycenter of the surviving crystals in the shifting box, as described in the previous section. The actual positions of the surviving crystal barycenters are obtained by adding the co-ordinates of the barycenters in the shifting box with the total shift of the simulation box as shown in Fig. 6 for three tracking veins. For calculating the general tracking efficiency, the expression proposed by Ankit et al. (2013b) is redefined for a small time interval $\delta t$ and averaged over the total simulation time, according to

$$
\mathrm{GTE}_{2}^{i}=\frac{\sum_{i=1}^{n(\delta t)} \frac{\theta_{\text {bary }}^{i}}{\theta_{\text {traj }}^{i}} \cdot(\delta t)}{n(\delta t)}
$$

If $\delta t$ is small, we rewrite the equation to

$$
\mathrm{GTE}_{2}=\frac{\int_{0}^{t} \frac{\theta_{\text {bary }}(t)}{\theta_{\text {traj }}(t)} \cdot \mathrm{d} t}{t}
$$

The values of $\mathrm{GTE}_{1}$ and $\mathrm{GTE}_{2}$ are calculated for the two simulations $A$ and $B$. The resulting values for $\mathrm{GTE}_{1}$ and $\mathrm{GTE}_{2}$ are listed in Table 2 and it can be seen that the associated temporal evolutions compare well. On the contrary, $\mathrm{GTE}_{1}^{t}$ and $\mathrm{GTE}_{2}^{t}$ derived from final microstructures, i.e. last simulation time-step differ significantly from $\mathrm{GTE}_{1}$ and $\mathrm{GTE}_{2}$ and show larger deviations with respect to each other. Additionally, the estimated $\mathrm{GTE}_{2}$ vary with axial tilt and therefore, may lead to erroneous interpretation.
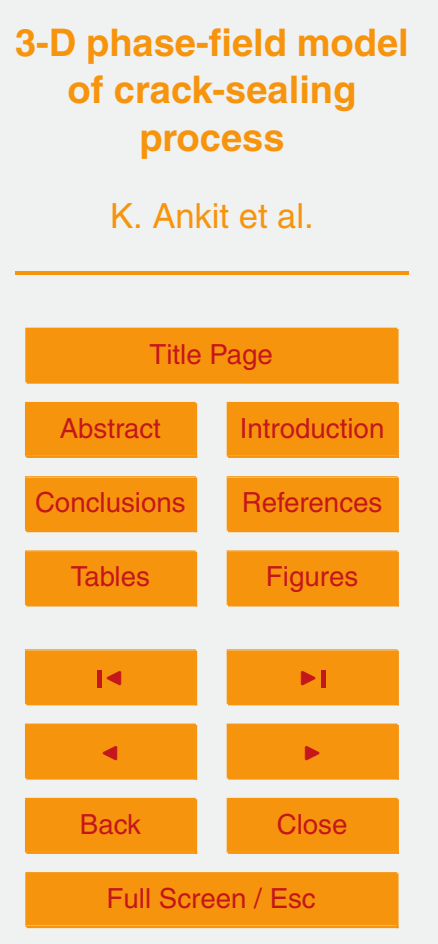

Printer-friendly Version

Interactive Discussion 


\subsection{Statistics}

It is well known from the 2-D numerical studies of crack-sealing process that a smaller crack-opening rate favors the formation of fibrous morphology, while, a larger crack opening rate leads to formation of blocky veins (Ankit et al., 2013b; Hilgers et al., 2001).

5 However, none of the previous studies focuses on the statistical aspects which have the potential to provide valuable insights into the vein growth process. We plot the number of grains surviving the crack opening process (in contact with the advancing crack surface) vs. the time dependent crack opening distance and observe a shift between the two regimes, in the present 3-D simulations. As shown in Fig. 7a, the decline in the number of grains is significantly steeper for faster crack opening (simulation $B$ ), as compared to the case of slow opening (simulation A). The plummeting of grain count is indicative of the anisotropy in surface energy being dominant, leading to orientation selection and growth competition, similar to free-growth conditions. In such a case, the mis-oriented grains are continuously eliminated by favorably oriented neighbors. On the contrary, when the crack-opening rate is smaller, the decrease in the number of grains is less steep and becomes constant, which indicates that grain boundaries are pinned by fractal peaks, even though the general tracking efficiency is near about 0.5 (much lesser than 1.0). The grain size distribution in the shifting box (final simulation timestep) is plotted in Fig. $7 \mathrm{~b}$ and represents those grains in contact with the advancing crack surface for the test cases $\mathrm{A}$ and $\mathrm{B}$. On comparison, it becomes clear that the tip of the distribution shifts towards smaller mean grain sizes due to an increased pinning behavior of fractal peaks when the crack-opening rate is smaller. It is noteworthy that a major repercussion of grain-boundary/multi-junction pinning as shown in Fig. 8 is that the growth competition based on mis-orientation is suppressed. In such cases, in Fig. 9. Therefore, a higher number of grains survive the crack-sealing process as opposed to other case when crack-opening rate is faster in evidence with the result in Fig. 7a.

GMDD

7, 631-658, 2014
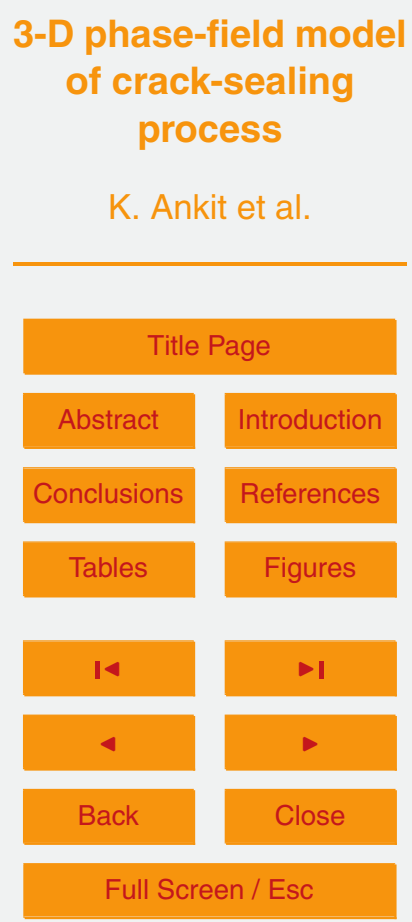

Printer-friendly Version

Interactive Discussion 


\section{Discussion of results}

The present work highlights the advantage of 3-D numerical studies over 2-D, by visualizing the complex and the inherently three-dimensional motion of grain boundaries/multi-junctions in crack-sealing process. In order to account for the tem-

5 poral evolution of veins, two different approaches to determine the general tracking efficiencies are proposed and compared. We clarify the main reason for the effect of the crack opening rate on the grain growth statistics, i.e. on the number of grains tracking the opening trajectory, size and orientation distribution.

For implementing a "realistic" 3-D fractal surface as the boundary condition and ob10 taining a uniform overlay of nuclei over the crack surface which prevents the onset of size-effects, preprocessing algorithms are proposed. An innovative approach to visualize the numerically simulated veins aided by post-processing techniques reveals that the grain boundary/multi-junction morphologies in 3-D are more complicated as compared to 2-D cases, in general. In order to deal with the third dimensionality which 15 makes the determination of tracking efficiency difficult, we amend the definition of general tracking efficiency to $\mathrm{GTE}_{1}$ and $\mathrm{GTE}_{2}$ given by Eqs. (4) and (6), respectively. While $\mathrm{GTE}_{1}$ may be interpreted as an extension of the tracking efficiency proposed by Urai et al. (1991) for the case of 3-D evolution, GTE $_{2}$ accounts for the temporal change in tracking behavior of veins with respect to the crack-opening trajectory. In contrast, $\mathrm{GTE}_{1}^{t}$ describes the tracking efficiency of the final microstructure (" $t$ " being the total simulation time) and does not account for temporal evolution. The study of peak trajectories demonstrates that $\mathrm{GTE}_{1}^{t}$ is significantly higher than the $\mathrm{GTE}_{1}$ for the simulation test cases, $A$ and $B$. It is noteworthy that $\mathrm{GTE}_{1}$ and $\mathrm{GTE}_{2}$, both accounting for the temporal evolution of grain boundaries/multi-junctions, compare quite well as 25 summarized in Table 2. The incongruousness arising out of the neglect of temporal evolution of grain boundaries/multi-junctions implies that conclusions about the tracking efficiency, as previously drawn upon the $\mathrm{GTE}_{1}^{t}$ data, which solely rely on final microstructure have a clearly limited validity. While the original approach of using grain
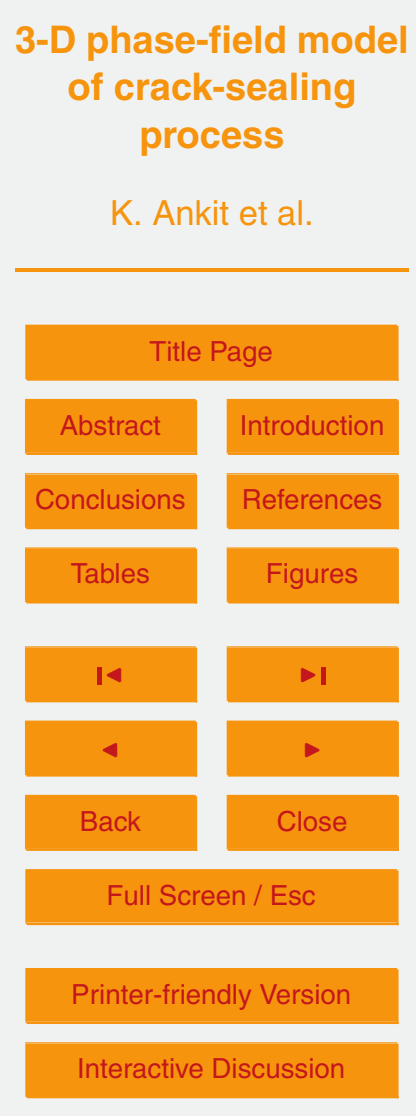

Interactive Discussion 
boundary morphology to determine tracking efficiency proves to be inept in 3-D, we conclude that the proposed GTEs, both accounting the temporal evolution, serve as reliable methods to quantify the tracking behavior in veins.

The grain evolution statistics obtained by post-processing the 3-D computational mi5 crostructure reveals that the number of grains in contact with advancing crack surface (Fig. 7a) decreases steeply when the crack-opening rate is higher. It can be argued that a steep decline in the number of grains and survival of crystals oriented along the most preferred orientation, is indicative of growth competition which does not relate to the boundary conditions namely the crack surface roughness or the opening rate. 10 The near incapability of fractal peaks to pin the grain boundaries/multi-junctions when crack-opening rate is higher can be seen in Fig. $5 \mathrm{c}$ and $\mathrm{d}$, where reddish grains are found in majority. On the contrary, the decline in the number of grains is less steep for smaller crack opening rate, which finally becomes constant. Such a conclusion on statistics can be attributed to stronger pinning of fractal peaks at grain boundaries and multi-junctions, which suppresses the growth competition based on mis-orientation of neighboring grains (Fig. 9). An interesting outcome of visualizing the temporal evolution in Fig. 8, is that the fractal peaks pin more strongly at quadruple junctions (grain multi-junctions in general) as compared to grain boundaries. The increase in pinning behavior of fractal peaks at smaller crack-opening rates is further accentuated by shift in the apex of grain size distribution towards small grain size (Fig. 7b). Thus, the plots of number of surviving grains as well as grain size distribution in Fig. 7 provide a statistical realization of the shift in regime and explain the vein characteristics observed in Fig. 9, which is primarily governed by crack-opening velocity in the present test cases.

Finally, it is worth clarifying that the present definitions of GTEs are formulated with an intention to indicate the importance of temporal evolution in determining the tracking efficiency. We do not rule out the possibility of other definitions, which may be equally capable to quantify tracking behavior in 3-D with high precision. Within the scope of current work, the prime motive is to highlight the gain in accuracy by accounting for temporal evolution in the well known methodologies, which strongly emphasizes the
GMDD

$7,631-658,2014$
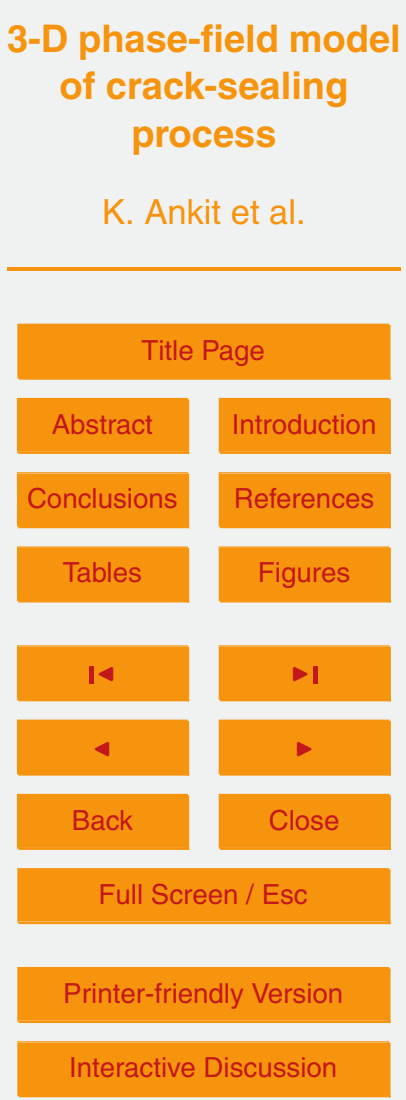
importance of 3-D numerical studies. The growth statistics, obtained by accounting for large number of grains and supplemented by 3-D visualization, aims to bridge the gap between field observations (of 3-D layers) and computational studies (limited to 2-D till date) and advances the understanding of the vein growth process.

\section{Conclusions and outlook}

The current work which is based on a thermodynamically consistent approach, namely the phase-field method aims to fill-in the short-comings while focusing on the aspects that have not been addressed in the previous numerical studies, chiefly the large-scale statistical studies as well as complex motion of grain boundaries/multi-junctions in 3-D. Wherever adequate, the benefits of 3-D simulations have been highlighted while enumerating the limitations of 2-D studies. The 3-D visualization aided by post-processing techniques supplement the present numerical studies and allow to draw meaningful conclusions from the simulation test cases. The most outstanding achievement of the present work is the characterization of vein microstructure based on temporal evolution 15 of grain boundaries/multi-junctions rather than relying on an approximate reconstruction from the final morphology, a popular approach in the geo-scientific community (Passchier and Trouw, 1996). Bearing in mind that it is fundamentally (essentially) difficult to design as well as carry out in-situ studies in laboratory experiments which can replicate the process of vein evolution, the 3-D numerical studies are of paramount importance as they can alternatively provide invaluable insights into the vein growth process. We further stress that the new methodologies based on multiphase-field modeling allow an efficient use of modern high super-computing power, so that even the consideration of large grain systems (up to 500000 grains) in 3-D computational studies becomes feasible.

25 Acknowledgements. K. Ankit and B. Nestler acknowledge the financial support by Graduate school 1483 of German Research Foundation and by the project CCMSE of the European Union (EFRE) together with the state Baden-Wuerttemberg.
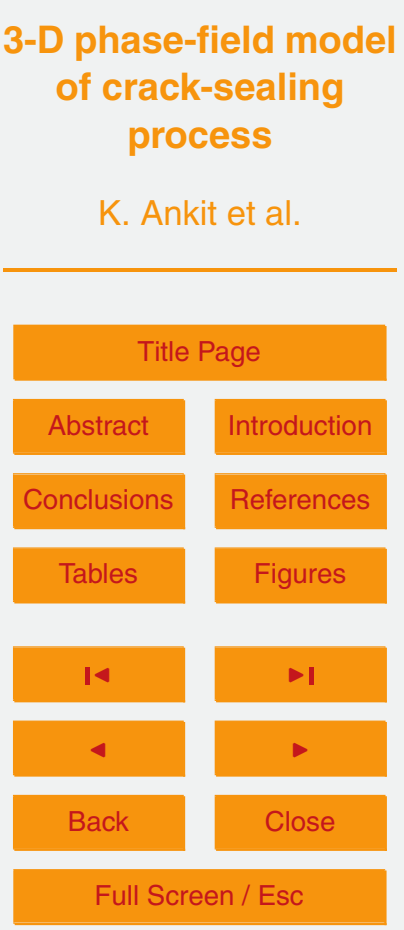

Printer-friendly Version

Interactive Discussion 


\section{References}

Ankit, K., Choudhury, A., Qin, C., Schulz, S., McDaniel, M., and Nestler, B.: Theoretical and numerical study of lamellar eutectoid growth influenced by volume diffusion, Acta Mater., 61, 4245-4253, 2013a. 638

5 Ankit, K., Nestler, B., Selzer, M., and Reichardt, M.: Phase-field study of grain boundary tracking behavior in crack-seal microstructures, Contrib. Mineral Petr., 166, 1709-1723, 2013b. 634, 636, 638, 640, 641

Bons, P.: Development of crystal morphology during unitaxial growth in a progressively widening vein: I. The numerical model, J. Struct. Geol., 23, 865-872, 2001. 633

Bons, P., Koehn, D., and Jessell, M. (Eds.): Microdynamics Modelling, Lecture Notes in Earth Sciences 106, Springer, Berlin, 2008. 633

Hilgers, C., Koehn, D., Bons, P., and Urai, J.: Development of crystal morphology during unitaxial growth in a progressively widening vein: II. Numerical simulations of the evolution of antitaxial fibrous veins, J. Struct. Geol., 23, 873-885, 2001. 641

15 Hubert, J., Emmerich, H., and Urai, J.: Modelling the evolution of vein microstructure with phase-field techniques - a first look, J. Metamorph. Geol., 27, 523-530, 2009. 634

Jessell, M., Bons, P., Evans, L., Barr, T., and Stüwe, K.: Elle: the numerical simulation of metamorphic and deformation microstructures, Comput. Geosci., 27, 17-30, 2001. 633

Miller, G.: Definition and rendering of terrain maps, in: Proceedings of the 13th annual conference on Computer graphics and interactive techniques (ACM), SIGGRAPH '86, New York, NY, USA, 39-48, 1986. 637

Nestler, B., Garcke, H., and Stinner, B.: Multicomponent alloy solidification: phase-field modeling and simulations, Phys. Rev. E, 71, 041609, doi:10.1103/PhysRevE.71.041609, 2005. 636

25 Passchier, C. and Trouw, R.: Microtectonics, 2nd Edn., Springer Verlag, Berlin, 1996. 644

Piazolo, S., Jessell, M., Bons, P., Evans, L., and Becker, J.: Numerical simulations of microstructures using the Elle platform: a modern research and teaching tool, J. Geol. Soc. India, 75, 110-127, 2010. 632

Stinner, B., Nestler, B., and Garcke, H.: A diffuse interface model for alloys with multiple components and Phases, SIAM J. Appl. Math., 64, 775-799, 2004. 636

Taylor, J. E. and Cahn, J. W.: Diffuse interfaces with sharp corners and facets: phase field models with strongly anisotropic surfaces, Physica D, 112, 381-411, 1998. 634
GMDD

$7,631-658,2014$

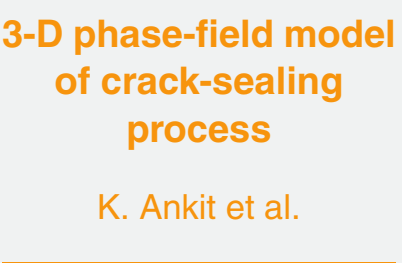

Title Page

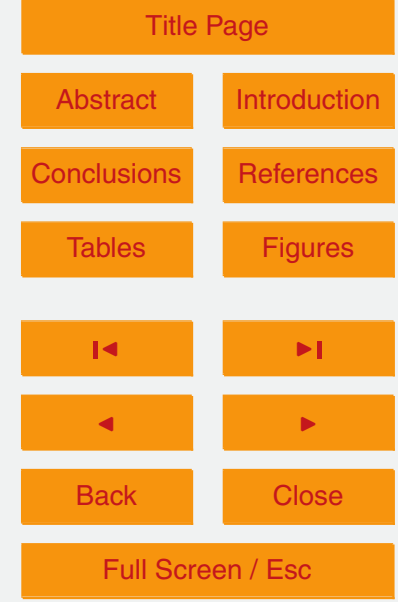

Printer-friendly Version

Interactive Discussion 
Urai, J., Williams, P., and van Roermund, H.: Kinematics of crystal growth in syntectonic fibrous veins, J. Struct. Geol., 13, 823-836, 1991. 638, 642

Wendler, F., Becker, J., Nestler, B., Bons, P., and Walte, N.: Phase-field simulations of partial melts in geological materials, Comput. Geosci., 35, 1907-1916, 2009. 636

5 Zhang, J. and Adams, J. B.: FACET: a novel model of simulation and visualization of polycrystalline thin film growth, Model. Simul. Mater. Sc., 10, 381-401, doi:10.1088/09650393/10/4/30, 2002. 633

K. Ankit et al.

Title Page

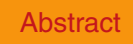

Introduction

Conclusions References

Tables

Figures

14

4

Back

Printer-friendly Version

Interactive Discussion 
Table 1. Table showing the choice of crack-opening rate for simulations $\mathrm{A}$ and $\mathrm{B}(\Delta x=\Delta y=\Delta z$ and $\Delta t=0.12$ ).

\begin{tabular}{cccc}
\hline Simulation & $\begin{array}{c}\text { Time between } \\
\text { successive opening }\end{array}$ & $\begin{array}{c}\text { Opening increment } \\
\text { in vertical direction }\end{array}$ & Trajectory \\
\hline A & $2 \Delta t$ & $3 \Delta x$ & Quarter arc \\
B & $2 \Delta t$ & $8 \Delta x$ & Quarter arc \\
\hline
\end{tabular}

3-D phase-field model

of crack-sealing

process

K. Ankit et al.

Title Page

Abstract

Introduction

Conclusions

References

Tables

Figures

14

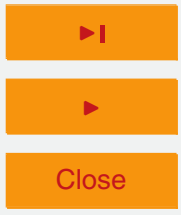

Back

Close

Full Screen / Esc

Printer-friendly Version

Interactive Discussion 
Table 2. Calculated general tracking efficiencies for the simulation test cases, $A$ and $B$. GTE 2 are comparable to the corresponding $\mathrm{GTE}_{1}$ whereas $\mathrm{GTE}_{2}^{t}$ and $\mathrm{GTE}_{2}^{t}$ show larger deviations. This indicates that the general tracking efficiencies compare quite well provided temporal evolution is accounted for its estimation.

\begin{tabular}{|c|c|c|c|c|}
\hline Axial tilt & $\mathrm{GTE}_{2}$ & $\mathrm{GTE}_{1}$ & $\mathrm{GTE}_{2}^{t}$ & $\mathrm{GTE}_{1}^{t}$ \\
\hline \multicolumn{5}{|c|}{ Simulation A } \\
\hline $5.63^{\circ}$ & 0.473 & \multirow{3}{*}{ 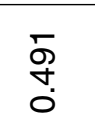 } & 0.234 & \multirow{3}{*}{$\begin{array}{l}0 \\
0 \\
0 \\
0\end{array}$} \\
\hline $42.45^{\circ}$ & 0.422 & & 0.751 & \\
\hline $58.5^{\circ}$ & 0.486 & & 0.639 & \\
\hline \multicolumn{5}{|c|}{ Simulation B } \\
\hline $5.63^{\circ}$ & 0.166 & \multirow{3}{*}{$\begin{array}{l}0 \\
\stackrel{\overbrace{}}{0} \\
0\end{array}$} & 0.167 & \multirow{3}{*}{$\begin{array}{l}\stackrel{\sim}{N} \\
\stackrel{\overbrace{}}{0}\end{array}$} \\
\hline $7.16^{\circ}$ & 0.204 & & 0.254 & \\
\hline $32.01^{\circ}$ & 0.196 & & 0.131 & \\
\hline
\end{tabular}

GMDD

7, 631-658, 2014

3-D phase-field model of crack-sealing

process

K. Ankit et al.

Title Page
Abstract

Conclusions

Tables

14

4

Back
Introduction

References

Figures

$\rightarrow$

$>$

Close
Full Screen / Esc

Printer-friendly Version

Interactive Discussion 

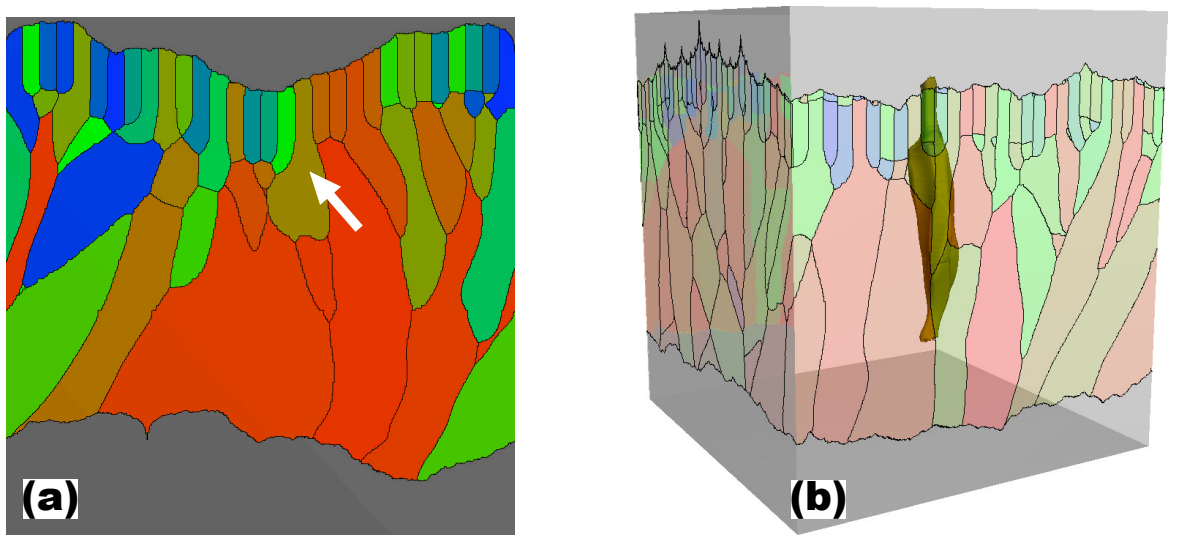

3-D phase-field model

of crack-sealing

process

K. Ankit et al.

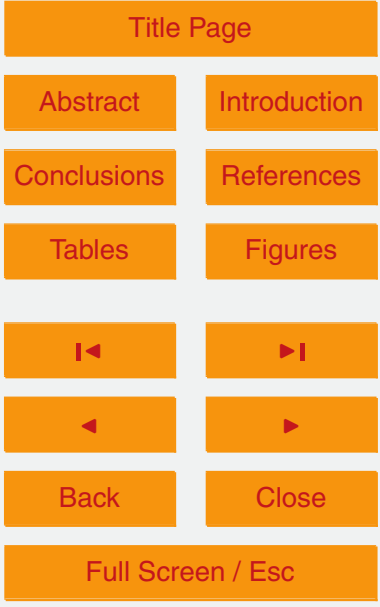

Printer-friendly Version

Fig. 1. Three dimensional phase-field simulation of the crack-seal microstructure. The direction of crack opening is along a quarter circle towards the left in the plane of computational thinsection. (a) Thin section cut of a 3-D computational microstructure showing the consumption of a grain as indicated by the white arrow. (b) The 3-D computational microstructure exposes that the grain was not consumed; rather it evolved along a different plane. Transparency of the surrounding grains enable the visualization of the tracking vein inside the numerical domain.

Interactive Discussion 

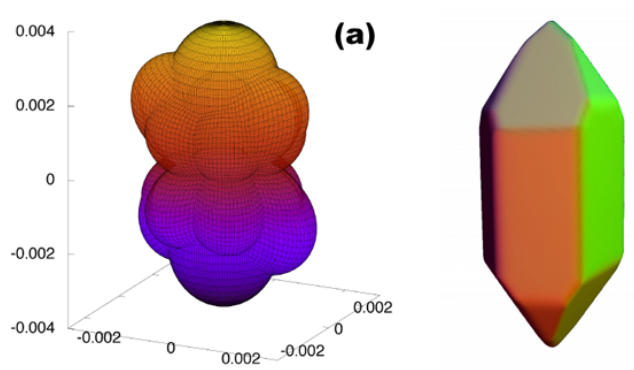

(b)

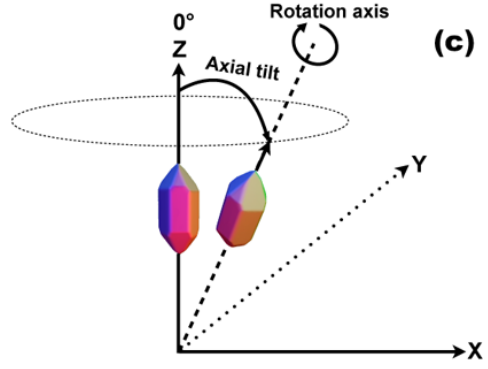

(c)

Fig. 2. (a) Polar plot of the interfacial energy for the symmetry of a quartz crystal. (b) Equilibrium quartz shape obtained from phase-field simulation. (c) The definition of crystal orientation in 3-D. The colors in (b) and (c) differentiate among crystal facets.
GMDD

7, 631-658, 2014

3-D phase-field model

of crack-sealing

process

K. Ankit et al.

Title Page

\section{Abstract}

Conclusions

Tables

14

4

Back
Introduction

References

Figures

I

$>$

Close
Full Screen / Esc

Printer-friendly Version

Interactive Discussion 

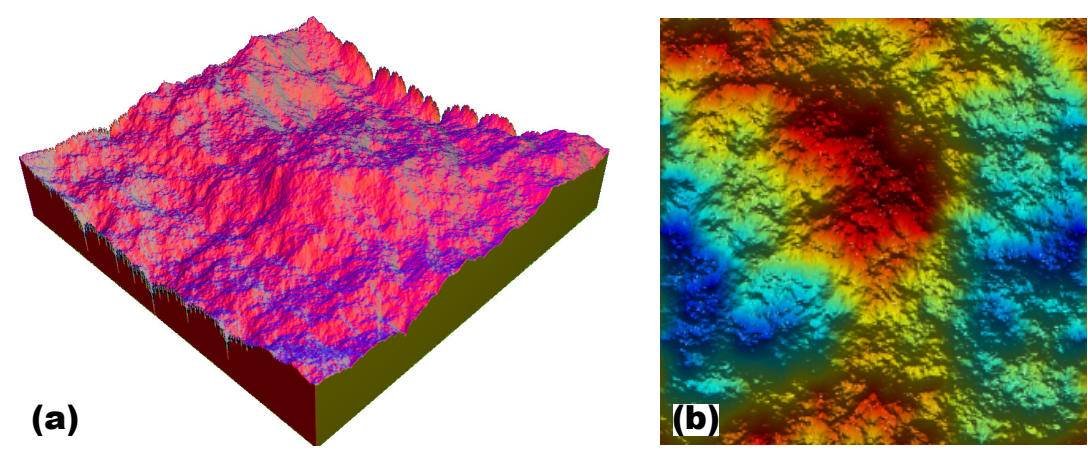

Fig. 3. (a) Fractal surface (generated by a well-known diamond-square algorithm) used for 3-D crack-sealing simulations. (b) Height-map of the generated surface.

\section{GMDD}

7, 631-658, 2014

3-D phase-field model of crack-sealing

process

K. Ankit et al.

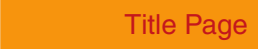

Abstract Introduction

Conclusions

References

Tables

Figures

14 $\rightarrow$

4

Back

Close

Full Screen / Esc

Printer-friendly Version

Interactive Discussion 


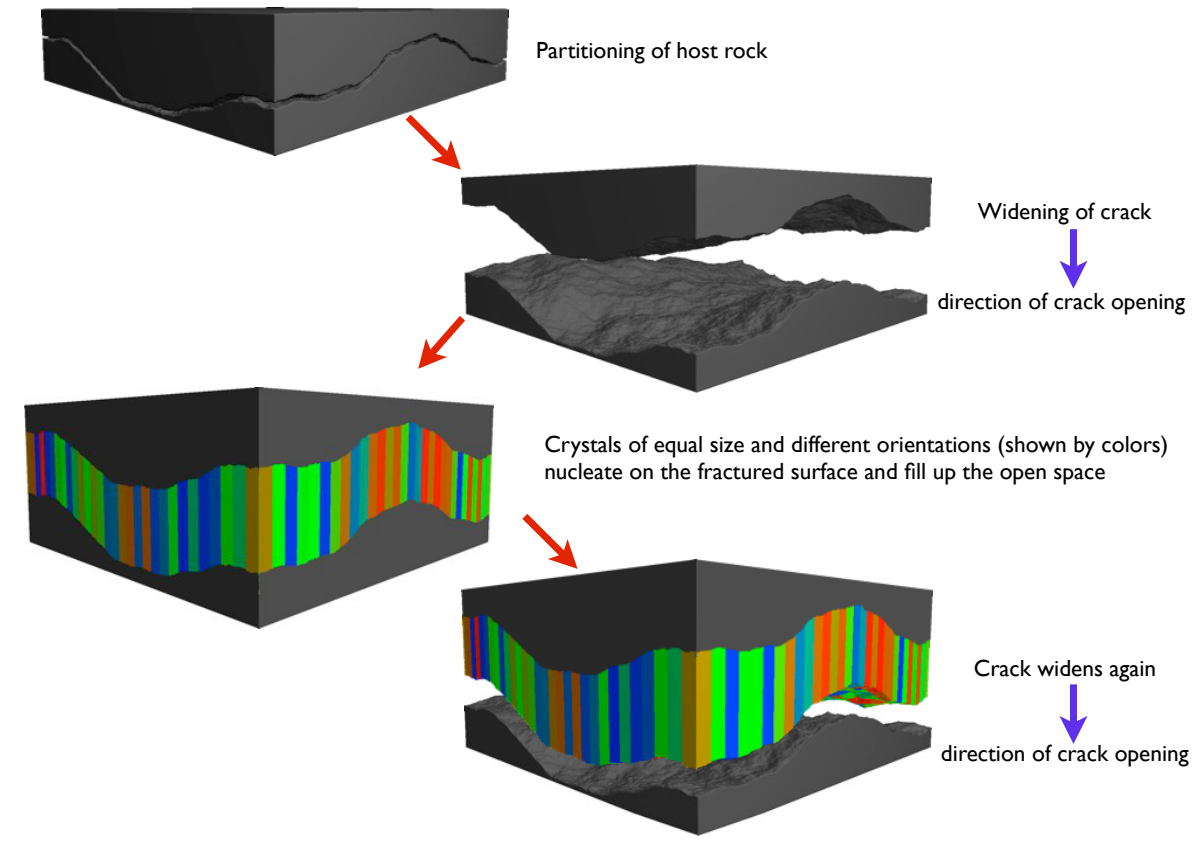

Fig. 4. Sequence of numerical pre-processing adopted to obtain a homogeneous overlay (of same size) of crystal nuclei on the algorithmically generated fractal surface. The final domain appearing in the above sequence is used as the initial condition for phase-field simulations.

3-D phase-field model of crack-sealing

process

K. Ankit et al.

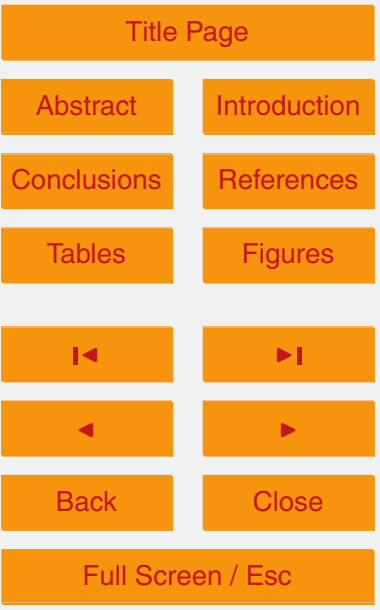

Printer-friendly Version

Interactive Discussion 

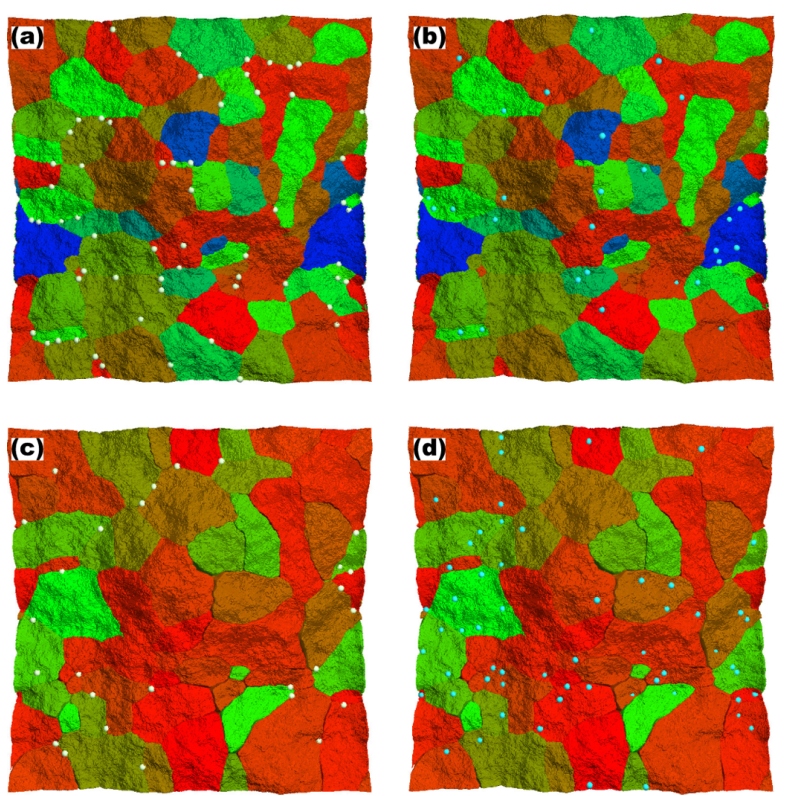

Tracking peaks

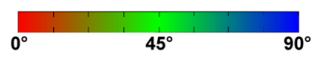

Fig. 5. Local peaks of crack surface (represented as colored spheres) plotted over the rockcrystal growth interface for simulation A in (a) and (b) and for simulation B as shown in (c) and (d). The fractal peaks tracking the grain boundaries/triple/quadruple junctions are plotted as offwhite spheres in (a) and (c). The fractal peaks not tracked by grain boundaries/multi-junctions are plotted as light-blue spheres in (b) and (d). On the basis of the final microstructures, the values of $\mathrm{GTE}_{1}^{t}$ (" $t$ " being total time) for simulation $A$ and $B$ are 0.685 and 0.325 respectively. On accounting for temporal evolution, the corresponding values depreciate to 0.491 and 0.206 . The grain colors refer to the axial tilt indexed in the color-bar.

3-D phase-field model of crack-sealing

process

K. Ankit et al.
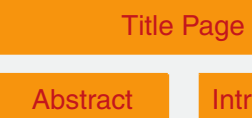

Introduction

Conclusions

References

Tables

Figures

14

-1

4

Back

$>$

Full Screen / Esc

Printer-friendly Version

Interactive Discussion
Close 

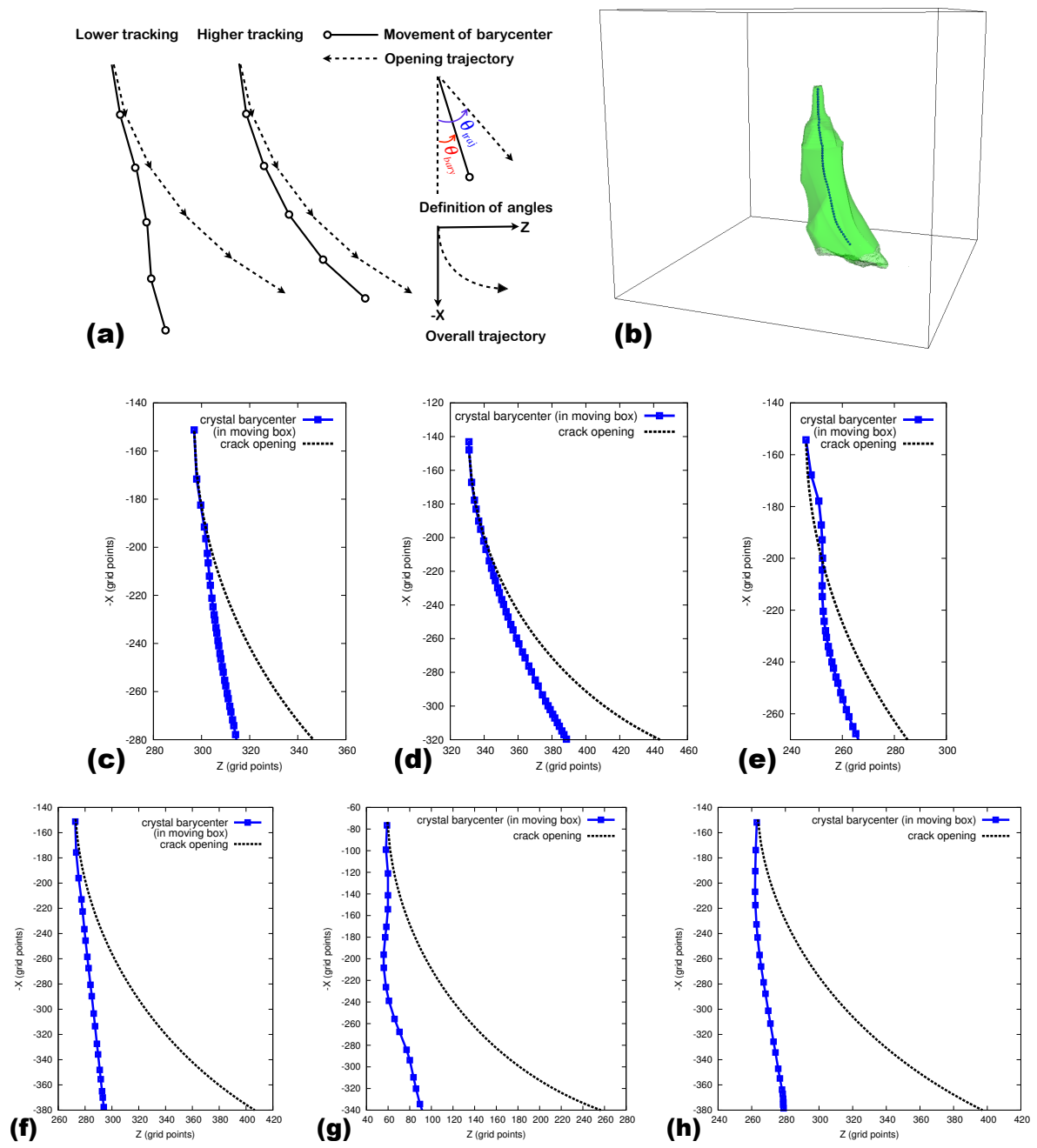

654

\section{GMDD}

7, 631-658, 2014

3-D phase-field model of crack-sealing process

K. Ankit et al.

\section{Title Page}

Abstract

Introduction

Conclusions

References

Tables

Figures

14

$>1$

4

Back

Close

\section{Full Screen / Esc}

Printer-friendly Version

Interactive Discussion 
Fig. 6. (a) Diagram explaining the calculation of $\mathrm{GTE}_{2}$ which accounts for temporal evolution of tracking efficiency. The solid line represent the evolution of the crystal barycenter in the shifting box while the dashed line corresponds to crack-opening trajectory. (b) In order to account for the time evolution in calculation of general tracking efficiency, the barycenter of surviving crystals (in contact with advancing crack surface) is determined. For the sake of better visualization, the barycenter of one of the surviving crystal is numerically masked over its iso-surface. The $\mathrm{GTE}_{2}$ is calculated for surviving crystals with axial tilts of (c) $5.63^{\circ}$ (d) $42.45^{\circ}$ and (e) $58.50^{\circ}$ in simulation $A$ and (f) $5.63^{\circ}$ (g) $7.16^{\circ}$ and (h) $32.01^{\circ}$ in simulation $B$.

\section{3-D phase-field model of crack-sealing \\ process \\ K. Ankit et al.}

Title Page

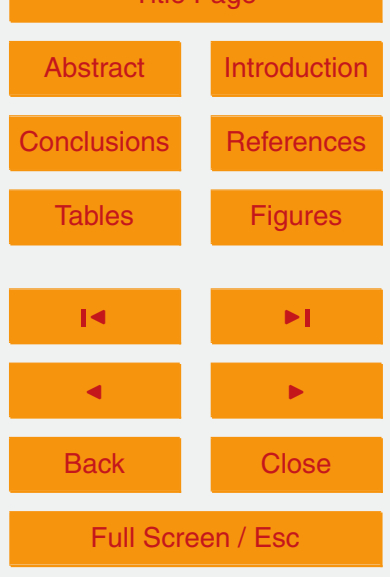

Printer-friendly Version

Interactive Discussion 

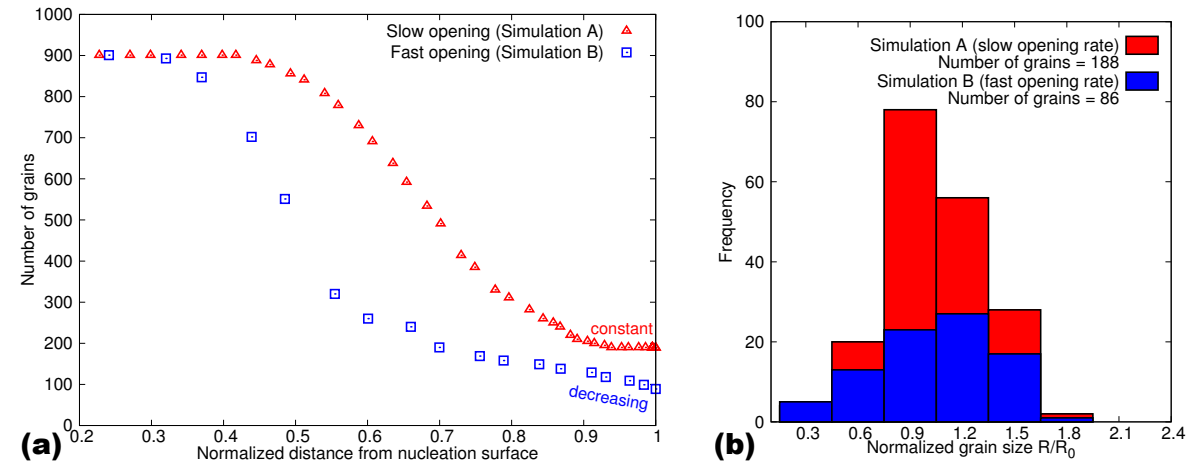

Fig. 7. Statistics obtained from 3-D phase-field simulations. (a) Number of grains in contact with the advancing crack surface plotted as function of normalized distance from the point of greatest depression on the nucleation surface. The number of grains become nearly constant when grain boundaries/triple points are pinned at facing peaks for small crack-opening rate. At higher crack-opening rates, the growth competition dominates due to a lesser "pinning effect" evident from a decreasing trend, even at later stages. (b) Grain size distribution for the final microstructures shown in Fig. 5. In the case of slower crack-opening, a shift of distribution peak towards smaller normalized grain size signifies greater pinning leading to higher grain boundary tracking efficiency.
GMDD

7, 631-658, 2014

3-D phase-field model of crack-sealing

process

K. Ankit et al.

Title Page

Abstract Introduction

Conclusions References

Tables

Figures

14 $\rightarrow 1$

4

Back

Close

Full Screen / Esc

Printer-friendly Version

Interactive Discussion 


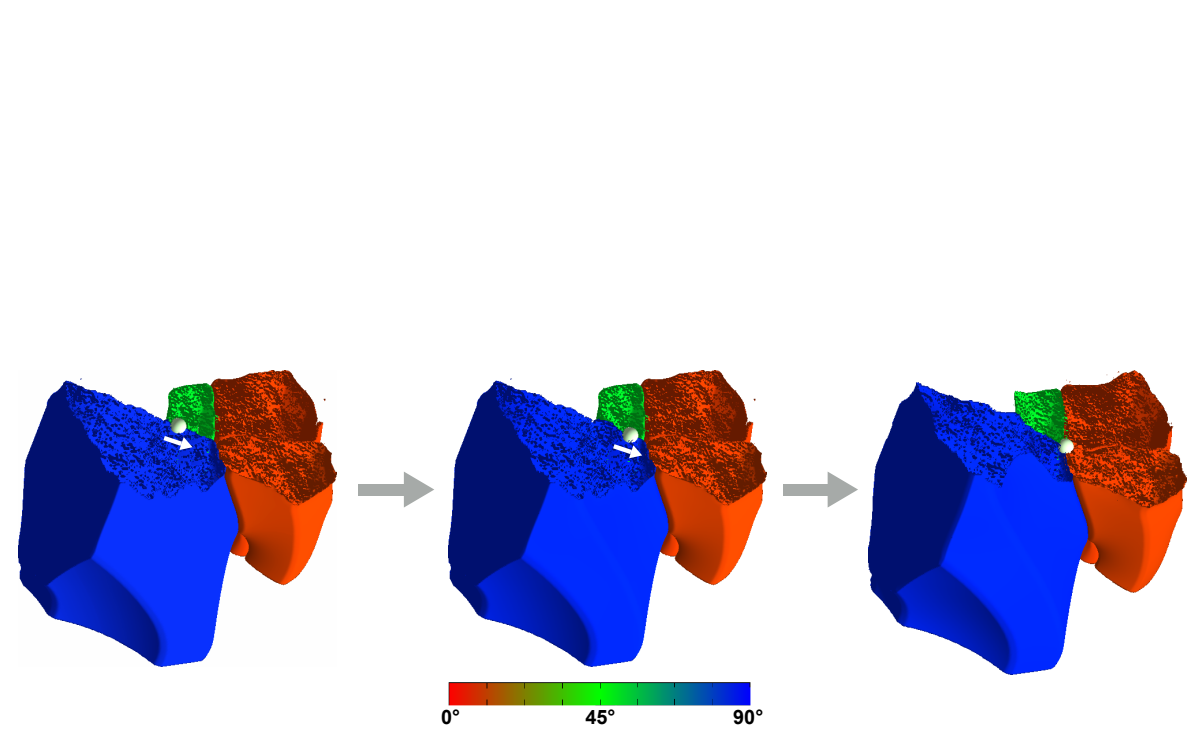

Fig. 8. Analysis of the temporal evolution of a fractal peak (in shifting box) which pins at the quadruple point and results in greater tracking behavior, as evident from survival of mis-oriented crystal fiber (in blue). The fractal peak illustrated as a white sphere acts as a grain boundary attractor while moving along a predetermined trajectory. It is interesting to note that the grain quadruple junction acts as a stronger attractor in comparison to grain boundaries, in the final stages of crack-sealing simulations (right image). The axial tilt of the grains are indexed according to the colorbar.

3-D phase-field model of crack-sealing process

K. Ankit et al.

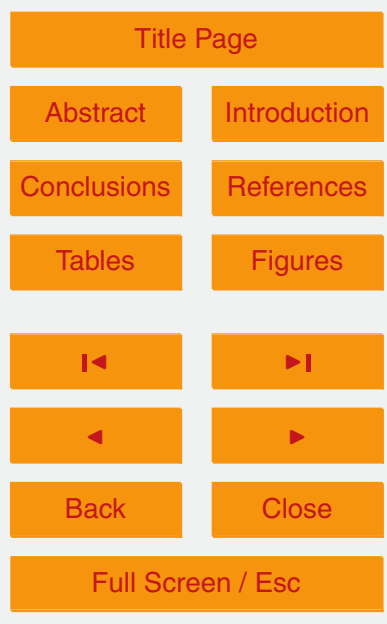

Printer-friendly Version

Interactive Discussion 


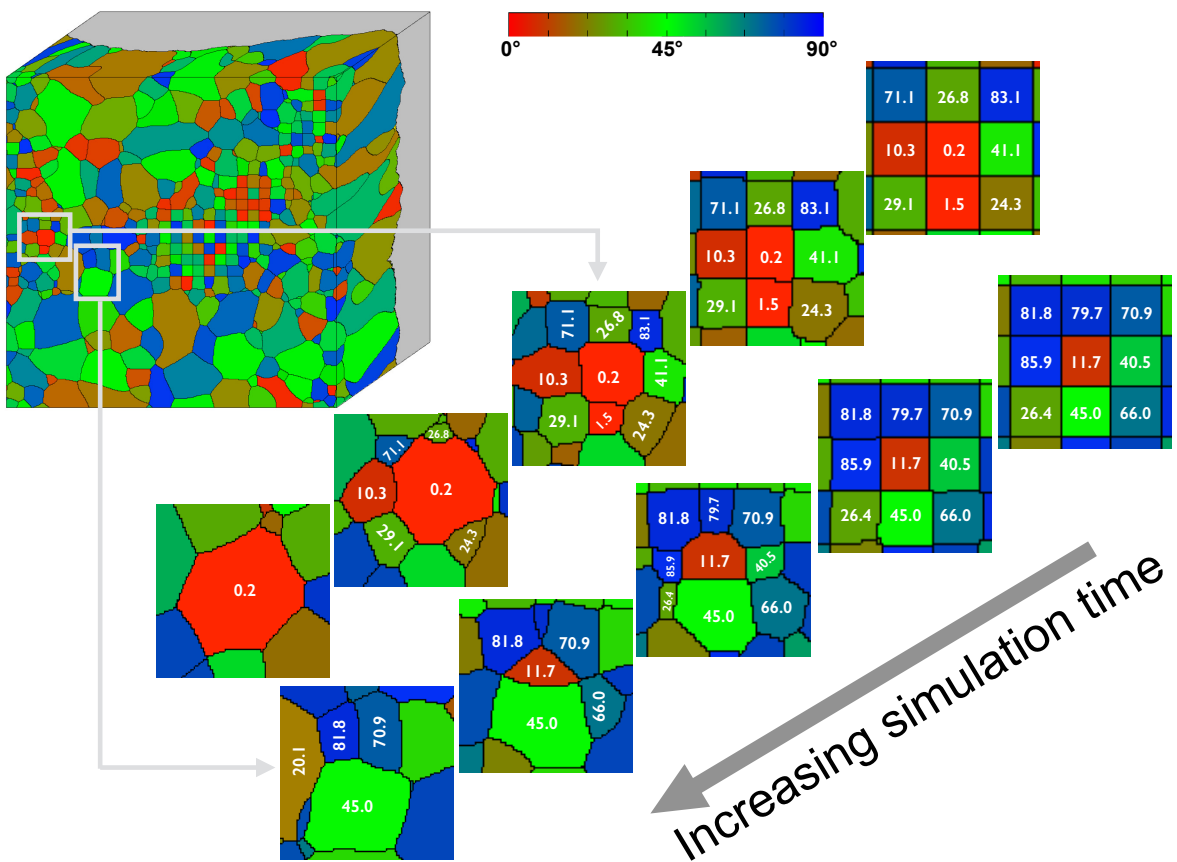

Fig. 9. Temporal evolution of marked areas in simulation A (slow crack-opening) show that the veins follow the trajectories of the opening peaks and evolve independently of their misorientation, with respect to the most preferred growth direction. In such a case, the grain boundaries/multi-junctions whose motion is pinned by the peaks of the advancing crack (shown in gray), track the opening trajectory. The color of the grains represent the axial tilt (numerical values also mentioned for grains in consideration) and indexed as per the colorbar.

\section{GMDD}

7, 631-658, 2014

3-D phase-field model of crack-sealing

process

K. Ankit et al.
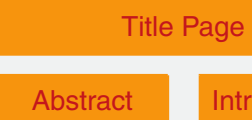

Introduction

Conclusions

References

Tables

Figures

14

$\rightarrow$

4

Back

Close

\section{Full Screen / Esc}

Printer-friendly Version

Interactive Discussion 